\title{
Definitive Radiotherapy in the Management of Non-Resectable or Residual Retroperitoneal Sarcomas: Institutional Cohort Analysis and Systematic Review
}

\author{
Aleksandra Sobiborowicz ${ }^{1,2, *}$, Mateusz Jacek Spałek, MD, PhD ${ }^{2, *} \mathbb{D}$, \\ Anna Małgorzata Czarnecka, MD, PhD, MSc $^{2,3}$, and Piotr Rutkowski, MD, PhD ${ }^{2}$
}

\begin{abstract}
Background: There is currently no consensus on optimal management of patients with primary or recurrent non-resectable/residual retroperitoneal sarcomas (RPS). The objective of this study was to document the outcomes of patients with primary or recurrent non-resectable/residual RPS treated in our center with definitive radiotherapy (RT) and to perform a systematic review on the topic.

Methods: A retrospective analysis of consecutive RPS patients treated in our center between 2000 and 2019 was performed. All consecutive patients who underwent definitive conformal RT with image guidance for primary or recurrent non-resectable or macroscopically residual RPS were included. Additionally, a systematic review compliant with the recommendations of the Preferred Reporting Items for Systematic Reviews and Meta-Analyses was performed.

Results: The study enrolled I 4 patients who met the aforementioned criteria. Data on clinicopathological characteristics, RT and response to treatment were assessed. RT allowed achieving prolonged local control of the disease, i.e. no local progression of the disease for more than 12 months after RT in 10 patients. Local control lasted more than 24 months in 6 cases, with minimal or no toxicity. A systemic review of II studies revealed concordance of our results with previous reports of primary or recurrent non-resectable/residual RPS.

Conclusions: RT provided satisfactory local disease control with acceptable treatment tolerance in patients with primary or recurrent non-resectable/residual RPS and represents a valuable treatment modality in the selected group of patients. Additional RT modalities i.e. BT, particle therapy, MRI-guided RT, or GRID/Lattice RT may be introduced to improve local control and further minimize toxicity.
\end{abstract}

\section{Keywords}

sarcoma, radiotherapy, retroperitoneal neoplasms, intensity-modulated radiotherapy, image-guided radiotherapy, abdominal neoplasms

Received July 15, 2020. Received revised November 06, 2020. Accepted for publication November 30, 2020.

\footnotetext{
' Laboratory of Experimental Medicine, Centre of New Technologies, University of Warsaw, Poland

${ }^{2}$ Department of Soft Tissue/Bone Sarcoma and Melanoma, Maria Sklodowska-Curie National Research Institute of Oncology, Warsaw, Poland

${ }^{3}$ Department of Experimental Pharmacology, Mossakowski Medical Research Centre, Polish Academy of Sciences, Warsaw, Poland

* Both author's contributed equally to this work.
}

Corresponding Author:

Mateusz Jacek Spałek, Department of Soft Tissue/Bone Sarcoma and Melanoma, Maria Sklodowska-Curie National Research Institute of Oncology, W.K. Roentgena 5, 02-78I Warsaw, Poland.

Email: mateusz.spalek@pib-nio.pl 


\section{Background}

Retroperitoneal sarcomas (RPS) - accounting for $15 \%$ of all soft tissue sarcomas (STS), are rare neoplasms with a general incidence rate in Europe of 0.31 per 100,000 people per year. ${ }^{1}$ The localization of RPS enables asymptomatic tumor growth to a large size with vital organ involvement and restricts surgical access, which make the management of RPS challenging and often with unsatisfactory results. ${ }^{2}$ Retrospective analysis of epidemiologic data from 45 European cancer registries showed 5-year relative survival rate of patients with RPS between 1995 and 2007 reached only $38.8 \%$ (95\% confidence interval 37.1-40.5). ${ }^{1}$ In a more recent analysis of 1007 patients treated at 2 North American and 6 European sarcoma centers, 5, 8, and 10 -year overall survival were $67 \%, 56 \%$ and $46 \%$, respectively, after a median follow-up of 58 months. ${ }^{3}$ Surgery is the primary treatment modality for RPS and microscopically radical (R0) resection is correlated with a decreased rate of abdominal recurrence and significantly longer survival. ${ }^{4-6}$ However, patients with gross residual disease after surgery (R2) seem to have no survival benefit from surgery compared to patients with tumors classified as non-resectable. ${ }^{5}$ Moreover, even when performed resection was optimal, the 5-year local control rate remains low, between $27 \%$ and $62 \%$, depending on the report. ${ }^{7}$ The most common cause for RPS treatment failure is local recurrence. In the aforementioned analysis of 1007 patients treated surgically for primary RPS, 316 of them developed local recurrence and in 249 cases it was the first sign of disease progression. ${ }^{3}$

Locally advanced, recurrent, or non-resectable RPS prompts administration of neoadjuvant and adjuvant therapies, including radiotherapy (RT) and chemotherapy; however, their role in the management of primary RPS remains uncertain. ${ }^{8,9}$ The use of RT in RPS is limited due to the large target volumes and a significant volume of adjacent radiosensitive organs at risk (OARs), such as the small bowel. Recently, the STRASS trial, a phase III randomized study of preoperative RT with surgery versus surgery alone for patients with primary RPS, failed to demonstrate a benefit of neoadjuvant RT in RPS management in the entire study population, and local control was improved in the liposarcoma subgroup only. ${ }^{10}$ However, in the STRASS trial, the recommended total dose (50.4 Gy) was lower than equivalent 2-Gy dose (EQD2) used in clinical practice or other RPS trials. Moreover, due to the late recurrence pattern in RPS, the primary endpoint of abdominal relapse-free survival and the secondary endpoint of overall survival require longer follow-up time to fully assess the effect of preoperative RT. Further, a study performed by the Trans-Atlantic Retroperitoneal Sarcoma Working Group (TARPSWG) showed no local control benefit of perioperative RT in RPS in multivariate analysis. ${ }^{4}$ By contrast, another retrospective analysis suggested improved local recurrence-free survival in the preoperative RT group in multivariate analysis $(\mathrm{p}=0.03) .{ }^{11}$ In a large retrospective Surveillance, Epidemiology, and End Results analysis, $20 \%$ improvement of overall survival in high-grade RPS was exhibited by adding postoperative RT. ${ }^{12}$ A metanalysis that compared RT versus no RT in various STS improved local control and overall survival in patients with RPS who underwent perioperative RT; however, analyzed studies were heterogeneous and did not contain any randomized clinical trial. ${ }^{13}$

Given there is currently no consensus on the optimal management of patients with primary or recurrent non-resectable/ residual RPS, contemporary conformal RT techniques with image guidance (IGRT), which has been an underestimated modality, could be valuable in this group of patients. In this retrospective cohort study, we aimed to document the outcomes of patients with primary or recurrent non-resectable/ residual RPS treated in our center with definitive IGRT. We also conducted a systematic review of the literature concerning this issue, to analyze the outcomes and safety profile of management of primary or recurrent non-resectable/residual RPS with RT.

\section{Methods}

\section{Analyzed Patient Group}

A retrospective analysis of consecutive RPS patients treated in the large tertiary sarcoma center between 2000 and 2019 was performed. We included all consecutive patients who underwent definitive IGRT for primary or recurrent non-resectable or macroscopically residual RPS. Extent of primary/recurrent/ residual disease, resectability, response to applied treatment according to the Response Evaluation Criteria in Solid Tumors 1.1 (RECIST) criteria and the Choi criteria, and indication for definitive RT were assessed by a sarcoma multidisciplinary tumor board that included surgical oncologists, radiation oncologists, medical oncologists, and radiologists. ${ }^{14} \mathrm{RT}$ was proposed to the patients with progressive disease after chemotherapy or during the observation period. Treatment of primary RPS was typical with surgery with or without chemotherapy (most often doxorubicin-based regimen). Pathological diagnoses were compliant with the WHO Classification of Tumours of Soft Tissue and Bone 4th Edition. ${ }^{15}$ All pathological diagnoses were centrally reviewed in our center by an experienced sarcoma pathologist.

\section{Radiotherapy}

IGRT was defined as RT techniques planned in 3 dimensions with image guidance (planar kilovoltage or cone beam computed tomography), namely 3-dimensional conformal RT (3D-RT), intensity-modulated RT (IMRT), volumetricmodulated arc therapy (VMAT), and stereotactic body RT (SBRT). SBRT was defined as RT with doses per fraction greater than 4 Gy and delivered in 5 or fewer fractions, prescribed to gross tumor volume (GTV) without elective margin. ${ }^{16}$ The following parameters were included in the analysis: pathology of the primary tumor, indication for RT, previous or concomitant systemic therapy, RT technique, total dose, EQD2, dose per fraction, target volumes, early and late RT toxicity, best local response, the incidence of local or distant relapse, date of disease progression, date of death (if 
applicable). Literature data suggest various, but often low alpha/beta ratio of sarcomas, mostly between 0.4 and 5 Gy. ${ }^{17}$ Thus, we assumed the alpha/beta ratio of sarcomas as 3 , to calculate EQD2. ${ }^{16}$

\section{Data Extraction}

Electronic medical records were screened with MedStream Designer software (Transition Technologies). Corresponding International Classification of Diseases code C48, C49, and keyword "radiotherapy" were used. All data were reviewed independently by 2 researchers.

\section{Statistical Analysis}

Descriptive statistics: as measures of frequency-count, percent, frequency were used; as measures of central tendencymean and median; and as measures of dispersion or variation range, variance and standard deviation. ${ }^{18}$ Toxicity was reassessed according to the Common Terminology Criteria for Adverse Events 5.0. The best local response was assessed using RECIST criteria and retrospectively reassessed using the Choi criteria by 2 authors. Prolonged local control was defined as no local disease progression at least for 12 months after IGRT. This was calculated as a difference in months between the RT and date of disease local progression (if occurred) or last follow-up. The Kaplan-Meier method for estimating survival functions was used. All $\mathrm{p}$ values $<0.05$ were considered significant. Missing data regarding the date of death were obtained from the National Cancer Registry. In cases of deaths from unknown reasons, the patients were treated as dead of disease progression. The evaluation of data was performed using the $\mathrm{R}$ software/environment, version 1.1.383, and jamovi project, version 1.6.3.

\section{Systematic Review}

The review was conducted according to the PRISMA (Preferred Reporting Items for Systematic Reviews and Meta-Analyses) guidelines. ${ }^{19}$ Online databases-PubMed, Scopus and Embase-were searched using the following formula of keywords: retroperitoneal AND sarcoma AND radiotherapy AND (non-resectable OR unresectable OR inoperable OR residual). Only full-text publications in English were included. There were no limits on the date of included publications. Included papers were original reports on definitive RT of primary or recurrent non-resectable or macroscopically residual RPS. Review articles and. publications that assessed RT as a neoor adjuvant treatment to surgery or only with palliative intent were excluded. The search of databases was supplemented with the "related articles" function, hand searches of reference lists of all available review articles, meta-analyses, original studies and handbooks. A review of the articles was performed by 2 of the authors independently, and any publications that were differentially classified were once again thoroughly evaluated based on inclusion criteria. Data concerning clinical characteristics of enrolled patients, RT techniques, total dose, dose per fraction, early and late RT toxicity, local control rates, and survival outcomes were then extracted by one of the authors with the accuracy checked by a second author.

\section{Results}

Between 2000 and 2019, 14 patients were treated for non-resectable/residual RPS with definitive IGRT in our center. Data on clinicopathological characteristics, RT, and response to treatment are summarized in Table 1. Eight of the 14 patients were male and the median age was 58 years (range: $29-71$ years). The most common pathologic diagnosis was leiomyosarcoma, which accounted for $42.8 \%$ of cases. Seven patients were treated for the recurrent non-resectable disease, 4 for primary non-resectable disease and 3 for residual disease. Among the patients who had received previous treatment, the majority had been treated with surgery and chemotherapy (most often dacarbazine and doxorubicin regimen). RT technique was IMRT/ VMAT in 11 out of 14 patients; whereas 3D-RT and SBRT were applied in 2 cases and one case, respectively. The median total dose used was 60.8 Gy (range: 30.0-66.0 Gy), median dose per fraction was 2.0 Gy (range: 1.8-5.0 Gy), and median EQD2 was 65.0 Gy (range: 46.0-80.0 Gy). Median gross tumor, clinical target, and planned target volumes were as follows: $142.5 \mathrm{~cm}^{3}$ (range: $9.0-550.0 \mathrm{~cm}^{3}$ ), $515.0 \mathrm{~cm}^{3}$ (range: $27.0-1144.0 \mathrm{~cm}^{3}$ ), and $652.5 \mathrm{~cm}^{3}$ (range: $41.0-1462.0 \mathrm{~cm}^{3}$ ). According to RECIST, the best response to treatment was a partial response in 6 patients, whereas 7 patients had a stable disease, and one patient progressed despite RT. According to the Choi criteria, the best response to treatment was also partial response, and it was observed in 9 patients, whereas a one patient had stable disease, and one patient progressed despite RT; for 3, patients imaging data were not available for reassessment using Choi criteria. Median follow-up was 24.1 months. Prolonged 12- and 24-month local control was observed in 10 and 6 patients, respectively. Median local control time was 27.7 months. Median overall survival was 41.5 months. Kaplan-Meier plots of local control and overall survival are presented in Supplement A and Supplement B, respectively. In an additional exploratory analysis, we did not find significant difference in local control between patients who received EQD2 63 Gy or more and less than EQD2 63 Gy ( $p=0.055$; see Supplement C). There were 4 events of early toxicity observed in 3 patients (two grade 1 gastrointestinal toxicities, one grade 1 skin toxicity, and one case of mild pain within the irradiated volume). Late toxicities occurred in 2 patients and manifested as grade 1 skin toxicity and persistent mild pain within the irradiated volume.

\section{Discussion}

In our study, contemporary IGRT allowed at least 12 months of local control in $71 \%$ of the patients. Moreover, local control lasted more than 24 months in 6 cases $(43 \%)$, indicating vast clinical benefits from RT. Data on the definitive treatment of 




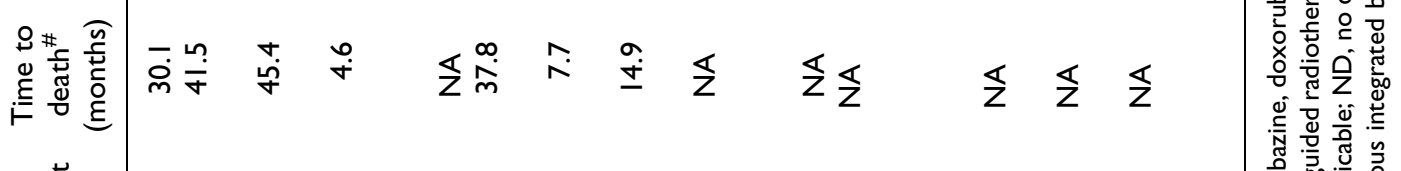

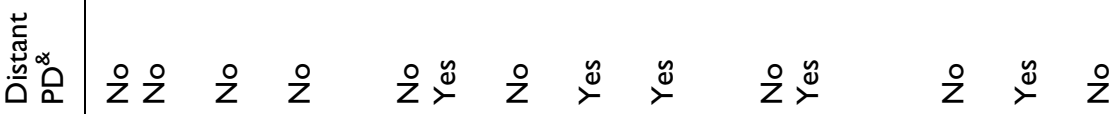

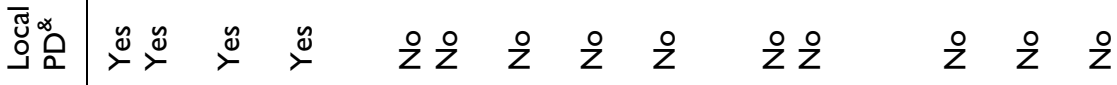

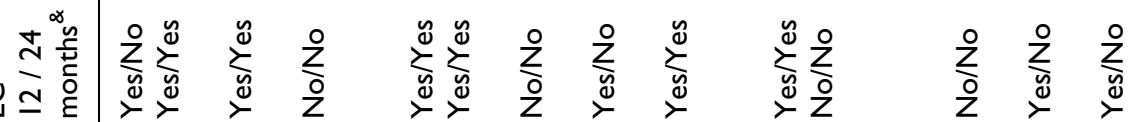

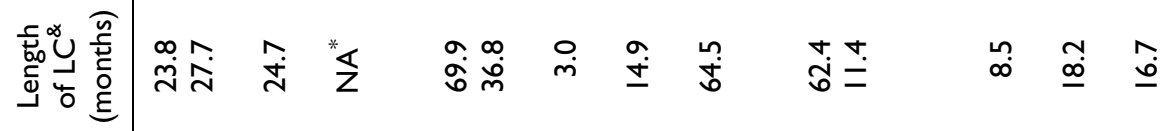





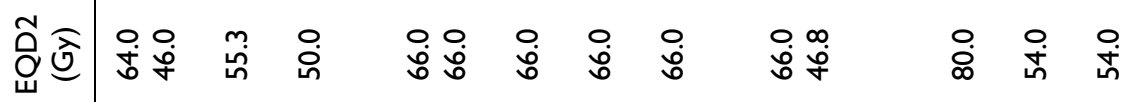



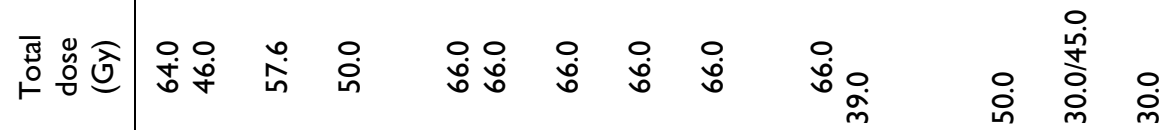

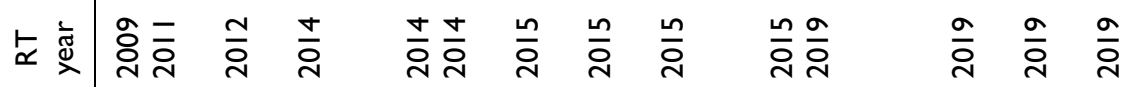

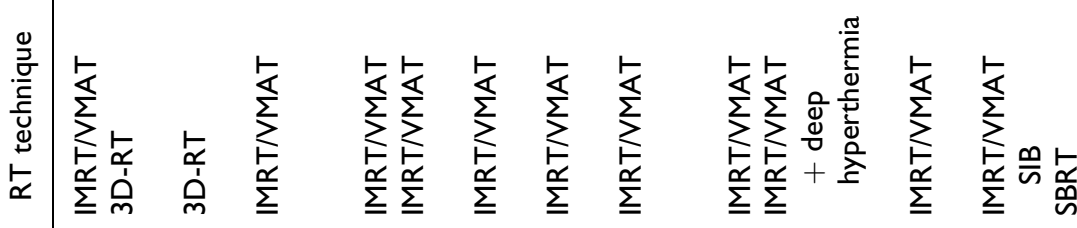

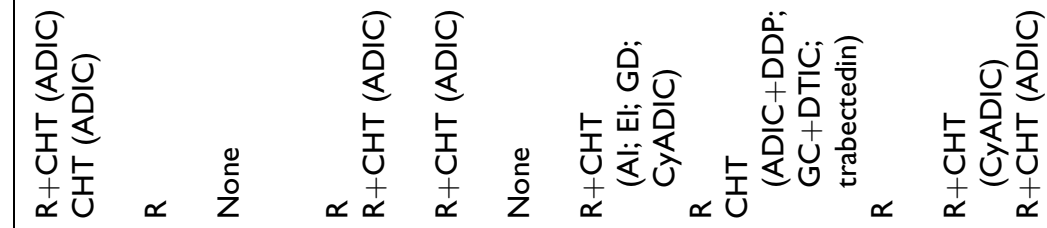



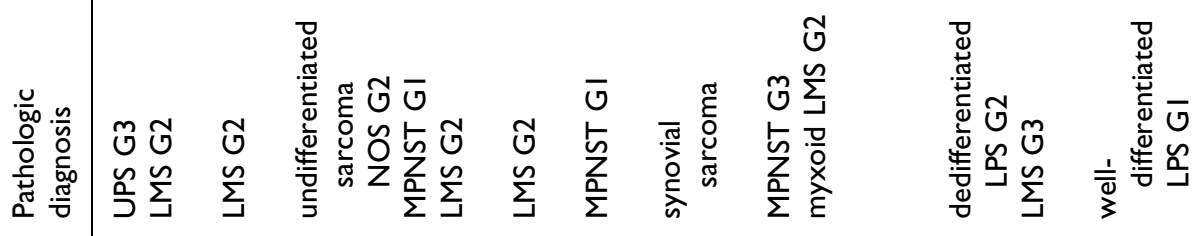

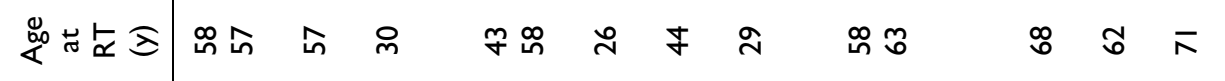






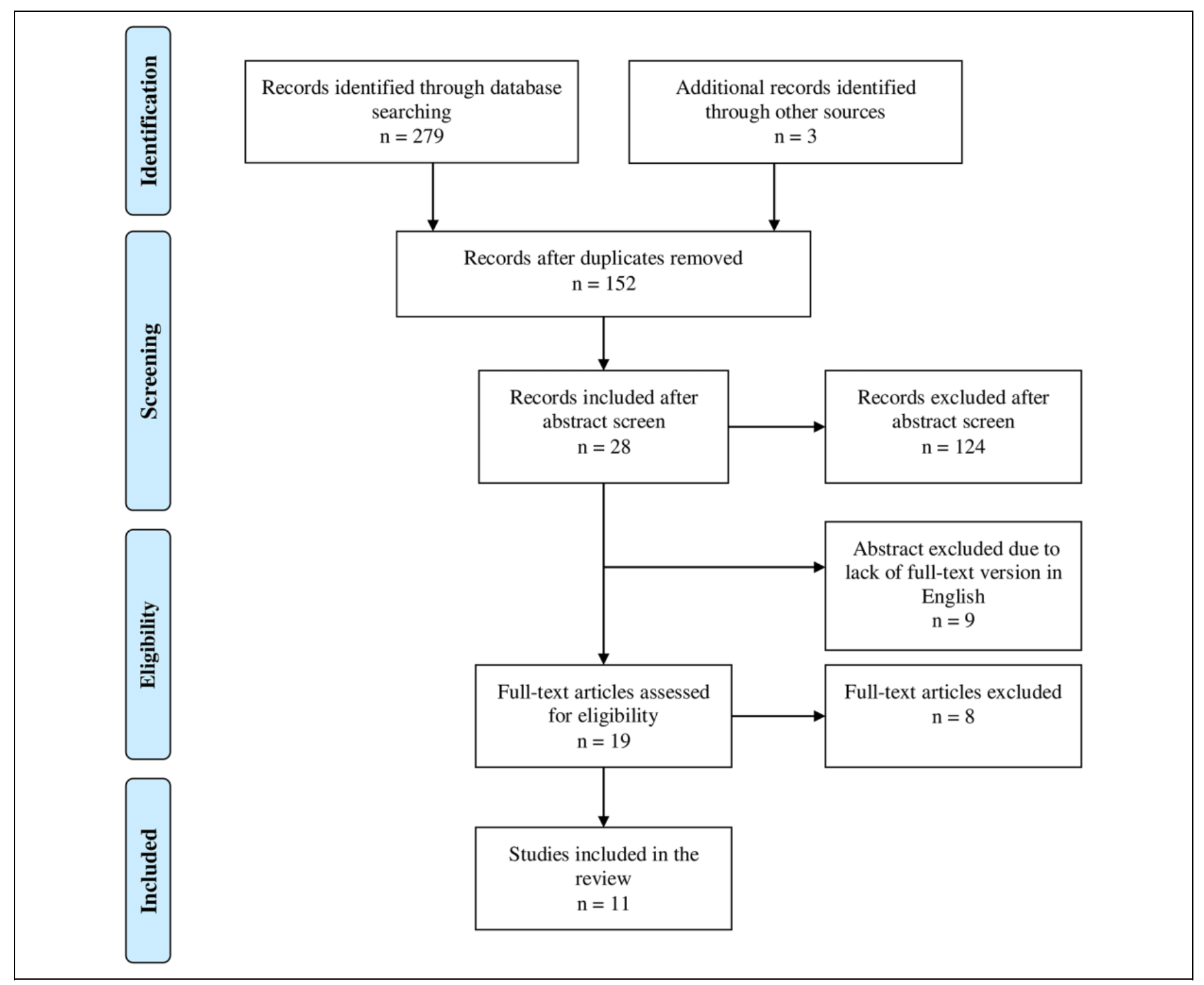

Figure I. PRISMA flow diagram.

non-resectable/residual RPS with photon-based RT remain greatly limited. In a retrospective analysis of 112 patients with unresectable STS, which included a subgroup of 29 patients with RPS, higher total doses were related to a better local control and overall survival in the entire cohort. ${ }^{20}$ In that study, a threshold of 63 Gy led to the highest statistical significance. We explored this threshold in our analysis showing no statistically significant difference at a cutoff of $63 \mathrm{~Gy}(\mathrm{p}=0.055)$, presumably due to the underpowered analysis. Nevertheless, in our cohort, there seemed to be a clinical benefit with EQD2 higher than 63 Gy with no local progression until $>20$ months. In a large retrospective analysis on patients with STS of different anatomic localizations treated with postoperative RT, doses of 64 Gy or above were associated with significantly improved rates of local control in comparison to doses in the order of $60 \mathrm{~Gy} .{ }^{21}$ In more radioresistant sarcomas, total doses $>65$ Gy and $>70$ Gy were associated with improved local control and overall survival. ${ }^{22,23}$
Delivery of high doses is substantially limited in RPS of large volumes and the proximity of OARs. However, recent developments in RT techniques have enabled the precise targeting of high doses to match tumor volumes, which greatly diminishes the involvement of OARs. ${ }^{24}$ This allowed for delivery of EQD2 $\geq 64 \mathrm{~Gy}$ in half of the patients included in our study. SBRT was possible in one patient whose GTV was relatively small. The use of IGRT in our series enabled good treatment tolerance with minimal acute toxicity — grade 1 acute toxicity developed only in 4 patients and higher-grade toxicities were not observed.

This is in concordance with reports included in our systemic review, the majority of which described mild or no cases with acute toxicities. Figure 1 shows the PRISMA flow diagram, documenting the number of search results, publications excluded after title/abstract review and full-text review, and the number of articles that met the inclusion criteria. A total of 11 studies were included in this review, and the extracted data are summarized in Table 2 and Table $3 .^{25-35}$ The PRISMA 


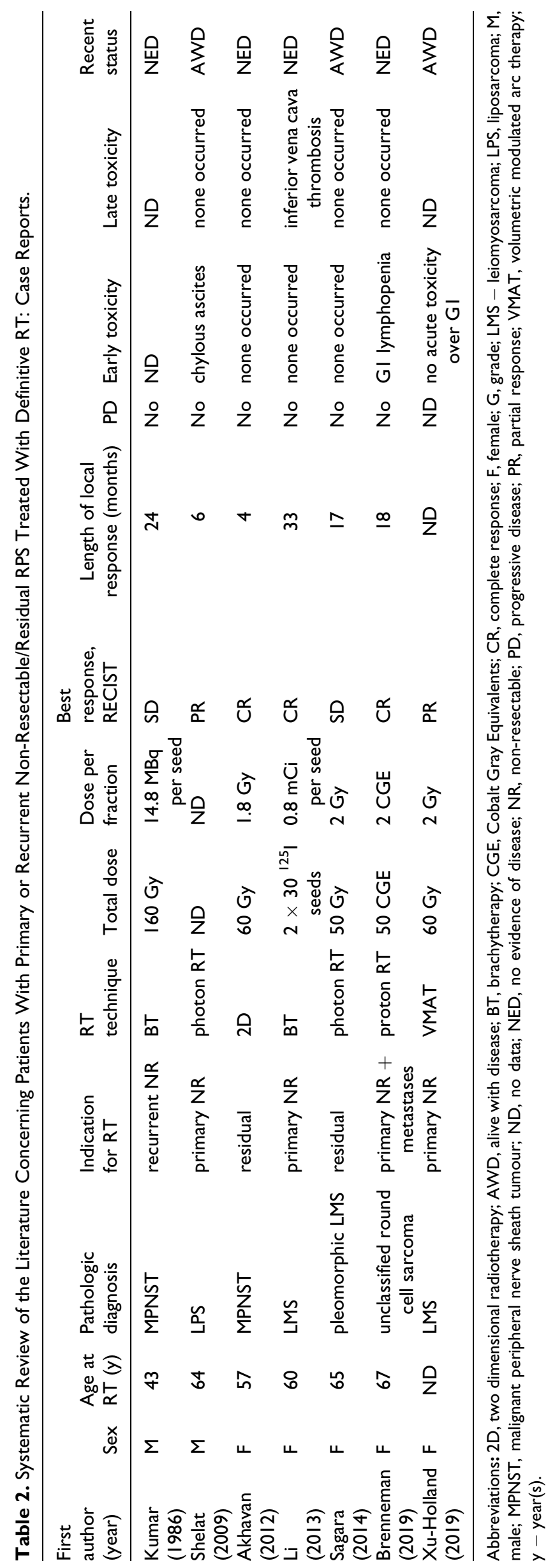




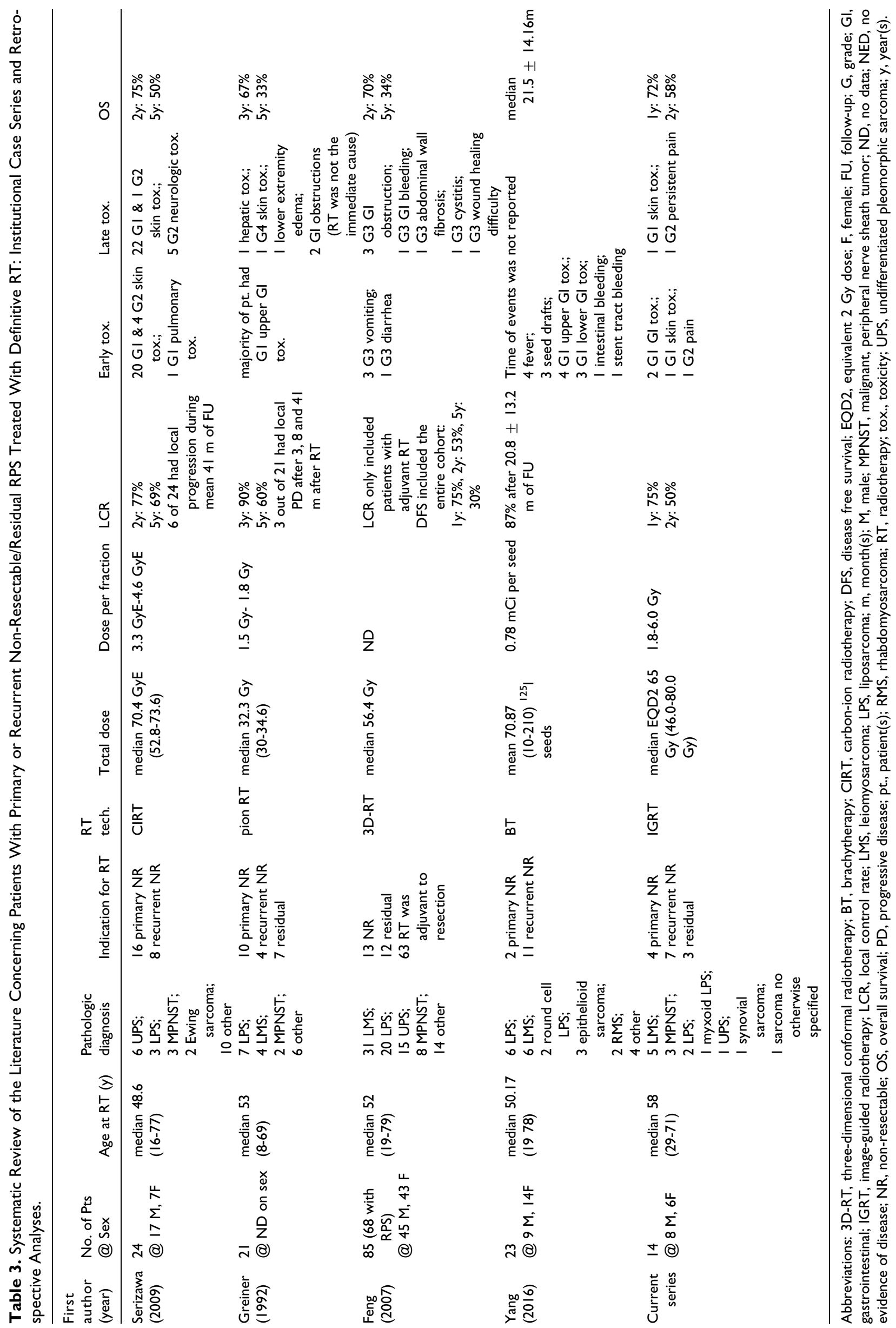


checklist is presented as Supplement D. In the reviewed articles, acute toxicity most commonly involved skin and gastrointestinal tract. Late toxicities, which in our report occurred only in 2 patients, were also mild and manageable. Although the late toxicities were rare, they typically involved the skin and nervous system. Greiner et al reported a case of grade 4 skin toxicity, and several grade 3 toxicities were reported by Feng et al. ${ }^{26,29}$ The occurrence of toxicities may be related to the total dose. A dose of 68 Gy or higher was related to the higher probability of severe toxicities, with $17 \%, 24 \%$, and $40 \%$ toxicity rate in the $\geq 68$ Gy group and $2 \%, 2 \%$, and $37 \%$, in the $<68$ Gy group at 2,5 , and 20 years, respectively $(\mathrm{p}=0.02)^{20}$

The rapid development of RT in the last decade urges the discussion of advances that may be useful in RPS management. First, MRI-guided RT allows for detailed imaging of RPS to accurately distinguish their borders from OARs. ${ }^{24}$ In a case report by Ghanem et al., a combination of MRI-guided RT and real-time MRI imaging enabled SBRT in the management of a small cell lung cancer metastasis to retroperitoneal space in close contact with the small bowel, achieving delivery of a dose of 27 Gy to the GTV in 3 fractions and durable near-complete response, and prevented acute gastrointestinal toxicity. ${ }^{36}$

A RT technique potentially useful in RPS management may be brachytherapy (BT), which was applied in 3 studies included in our systematic review. In 23 patients with non-resectable RPS treated with CT-guided ${ }^{125}$ I implantation as the only treatment modality, BT seemed safe and efficient, causing mild side effects. ${ }^{28}$ A significant decrease of visual analog scale score and objective response was achieved in all treated patients, with 3 local recurrences during the mean of 20.87 months follow up. Furthermore, there were 2 case reports on the use of BT in RPS, in which long-lasting complete responses were achieved. ${ }^{27,32}$ However, other reports described significant toxicities after intraabdominal BT. ${ }^{37} \mathrm{BT}$ toxicity profile is different than in external beam RT. RPS treatment with BT increases risks of neuropathy, hydronephrosis, fistulas, wound complications, and intraabdominal abscesses. Use of tissue expanders is recommended to provide spacing for OARs and avoid high doses in upper abdomen. Full recommendations of BT in RPS are provided in the consensus published by the American Society of Brachytherapy. ${ }^{37}$

Recent decades brought the development of RT techniques that use protons and heavy ions. The dose of particle-based RT is expressed in Gray-equivalents (GyE), calculated as carbon physical dose in Gy multiplied by Relative Biological Effectiveness (RBE), which in the case of carbon ions was empirically determined as equal 2.5 to 3 , whereas protons are regarded as having RBE of 1.1. In a case series by Yoon et al. (2010) preoperative proton-beam radiation therapy in RPS was shown to allow marked sparing of OARs in comparison to IMRT. ${ }^{38}$ In a prospective, phase I clinical trial of preoperative intensity-modulated proton therapy for RPS, patients received 50.4 GyE in 28 fractions in tumor volume and clinical margin with a simultaneous integrated boost in tumor to doses of 60.2, 61.6, and 63.0 GyE in 28 fractions. Only mild acute toxicities and one late grade 3 hydronephrosis were observed. ${ }^{39}$ However, the results of a parallel phase I study of the IMRT have not yet been published. Two studies included in our systematic review described the efficacy of particle RT in the setting of unresectable RPS. ${ }^{25,34}$ A case report by Brenneman et al. (2019) described a patient with metastatic RPS treated with proton beam RT, resulting in a near-complete response of the primary lesion and complete regression of all metastases. ${ }^{34}$ Serizawa et al. (2009) published a case series of 24 patients treated with carbon ion RT (CIRT) for non-resectable RPS. ${ }^{25}$ CIRT allowed for relatively high local disease control and satisfactory survival outcomes, with manageable toxicities, as most patients developed only grade $\leq 2$ skin acute reactions. In a retrospective analysis by Imai et al. (2018), CIRT reportedly allowed high irradiation doses with mild toxicity and satisfactory local control in non-resectable axial STS. ${ }^{40}$ CIRT may also prove useful in the management of recurrent sarcomas in anatomical localizations that hinder surgical access, which can result in functional or aesthetic damage, such as the orbit and spermatic cord or pelvis and the spine. ${ }^{41,42}$ However, CIRT is still an experimental technique, with potentially unknown late complications and it should not be directly compared to photon RT.

Another approach could be the combination of concomitant or interdigitated chemotherapy with RT. Preliminary results of such regimens are promising. For example, definitive RT (60 Gy) combined with single-agent ifosfamide in patients with unresectable STS led to 70\% 5-year control; however, the main reason for treatment failure was metastatic spread. ${ }^{43}$ In a prospective phase II clinical trial, hypofractionated RT was combined with anthracycline-ifosfamide chemotherapy, giving promising results in bulky, marginally resectable STS of the extremities and trunk wall. ${ }^{44}$ However, such combinations have never been investigated in RPS. Thus, a prospective clinical trial is desired.

Advanced RT delivery and planning techniques resulted in the development of several approaches to the treatment of bulky tumors, such as spatially fractionated radiation therapy applied through sieve-like collimators, a so-called GRID therapy. ${ }^{45}$ GRID further evolved into 3-D lattice RT that restricts the high-dose regions to tumor volume. ${ }^{46}$ This allows for the delivery of doses in the range of 20 Gy to the tumor, with acceptable toxicities - its grid-like pattern of affected and non-affected tissues results in faster rate of regeneration. In several case reports, lattice RT enabled durable local control with tolerable side-effects in the management of large abdominal metastatic masses of gynecological neoplasms. ${ }^{47,48}$ GRID combined with ifosfamide showed potential efficacy in the treatment of extremity STS, suggesting that these techniques may prove useful in the management of large RPS. ${ }^{49}$ However, GRID is still an experimental technique and has not been approved for use beyond prospective clinical trials.

Some limitations of this study include its retrospective nature that might introduce selection bias. This also poses a significant risk of incomplete or misinterpreted data. To reduce 
the risk of potential bias, all available records were reviewed independently by 2 of the co-authors. Another weakness of the study is the lack of primary assessment of treatment response using Choi or EORTC STBSG criteria. Nevertheless, the retrospective reassessment of the response based on the Choi criteria was provided. In a planned prospective clinical trial with definitive RT for non-resectable RT after chemotherapy, we will use advanced diffusion-weighted MRI techniques to assess the response to treatment. Further, the study is limited by its relatively small sample size that does not allow for univariate, multivariate, and Cox regression analysis of the impact of selected factors on tumor response or local control. Further prospective studies on predictive factors may be useful in determining the ideal candidates for definitive RT in RPS. Nonetheless, this study and the systematic review provide valuable data, which is lacking due to the rarity of RPS and limited available publications on the role of contemporary RT techniques in patients with primary or recurrent non-resectable/ residual RPS.

\section{Conclusions}

Contemporary RT enables efficient local disease control with acceptable treatment tolerance in patients with primary or recurrent non-resectable/residual RPS. RT represents a valuable treatment modality in this selected group of patients. Additional RT modalities, including BT, particle therapy, MRI-guided RT, or GRID/Lattice RT, may be introduced in selected patients to improve local control and minimize toxicity.

\section{Authors' Note}

All data generated or analyzed during this study are included in this published article (in the tables). MS conceived of the presented idea. AS and MS retrieved data. AS and MS performed the literature review. AS and MS prepared the draft. AMC and PR performed quality control. AMC and PR provided critical revision of the article. MS, AS, AMC and PR discussed the concerns and contributed to the final manuscript. Ethical review process was not required due to the retrospective design of study and anonymized data of patients included, in accordance with the local legislation and institutional requirements.

\section{Declaration of Conflicting Interests}

The author(s) declared no potential conflicts of interest with respect to the research, authorship, and/or publication of this article.

\section{Funding}

The author(s) disclosed receipt of the following financial support for the research, authorship, and/or publication of this article: This research did not receive any specific grant from funding agencies in the public, commercial, or not-for-profit sectors. The processing charges cost was covered by the Maria Sklodowska-Curie National Research Institute of Oncology, Warsaw, Poland.

\section{ORCID iD}

Mateusz Jacek Spałek, MD, PhD D https://orcid.org/0000-0002-3 960-7673

\section{Supplemental Material}

Supplemental material for this article is available online.

\section{References}

1. Gatta G, Capocaccia R, Botta L, et al. Burden and centralised treatment in Europe of rare tumours: results of RARECAREnet-a population-based study. Lancet Oncol. 2017;18(8): 1022-1039. doi:10.1016/S1470-2045(17)30445-X

2. Rutkowski P. Current therapy of retroperitoneal sarcomas. Oncol Clin Pract. 2018;14(6):348-353. doi:10.5603/OCP.2018.0048

3. Gronchi A, Strauss DC, Miceli R, et al. Variability in patterns of recurrence after resection of primary retroperitoneal sarcoma (RPS): a report on 1007 patients from the Multi-Institutional Collaborative RPS Working Group. Ann Surg. 2016;263(5): 1002-1009. doi:10.1097/SLA.0000000000001447

4. Bonvalot S, Rivoire M, Castaing M, et al. Primary retroperitoneal sarcomas: a multivariate analysis of surgical factors associated with local control. J Clin Oncol. 2009;27(1):31-37. doi:10.1200/ JCO.2008.18.0802

5. Kirane A, Crago AM. The importance of surgical margins in retroperitoneal sarcoma. J Surg Oncol. 2016;113(3):270-276. doi:10.1002/jso.24135

6. Raut CP, Callegaro D, Miceli R, et al. Predicting survival in patients undergoing resection for locally recurrent retroperitoneal sarcoma: a study and novel nomogram from TARPSWG. Clin Cancer Res. 2019;25(8):2664-2671. doi:10.1158/1078-0432. CCR-18-2700

7. Van De Voorde L, Delrue L, van Eijkeren M, De Meerleer G. Radiotherapy and surgery - an indispensable duo in the treatment of retroperitoneal sarcoma. Cancer. 2011;117(19):4355-4364. doi:10.1002/cncr.26071

8. Gronchi A, De Paoli A, Dani C, et al. Preoperative chemoradiation therapy for localised retroperitoneal sarcoma: a phase I-II study from the Italian Sarcoma Group. Eur J Cancer. 2014; 50(4):784-792. doi:10.1016/j.ejca.2013.11.021

9. Haas RL, Baldini EH, Chung PW, van Coevorden F, DeLaney TF. Radiation therapy in retroperitoneal sarcoma management. J Surg Oncol. 2018;117(1):93-98. doi:10.1002/jso.24892

10. Bonvalot S, Gronchi A, Péchoux CL, et al. Preoperative radiotherapy plus surgery versus surgery alone for patients with primary retroperitoneal sarcoma (EORTC-62092: STRASS): a multicentre, open-label, randomised, phase 3 trial. Lancet Oncol. 2020;21(10):1366-1377. doi:10.1016/S1470-2045(20)30446-0

11. Kelly KJ, Yoon SS, Kuk D, et al. Comparison of perioperative radiation therapy and surgery versus surgery alone in 204 patients with primary retroperitoneal sarcoma: a retrospective twoinstitution study. Ann Surg. 2015;262(1):156-162. doi:10.1097/ SLA.0000000000001063

12. Bates JE, Dhakal S, Mazloom A, Constine LS. The benefit of adjuvant radiotherapy in high-grade nonmetastatic retroperitoneal 
soft tissue sarcoma: A SEER analysis. Am J Clin Oncol. 2018; 41(3):274-279. doi:10.1097/COC.0000000000000259

13. Albertsmeier M, Rauch A, Roeder F, et al. External beam radiation therapy for resectable soft tissue sarcoma: a systematic review and meta-analysis. Ann Surg Oncol. 2018;25(3): 754-767. doi:10.1245/s10434-017-6081-2

14. Stacchiotti S, Collini P, Messina A, et al. High-grade soft-tissue sarcomas: tumor response assessment-pilot study to assess the correlation between radiologic and pathologic response by using RECIST and Choi criteria. Radiology. 2009;251(2):447-456. doi:10.1148/radiol.2512081403

15. WHO Classification of Tumours of Soft Tissue and Bone. Fourth Edition-WHO-OMS (2013). Accessed May 2, 2020. https:// apps.who.int/bookorders/anglais/detart 1 .jsp? codlan $=1 \&$ codcol $=70 \& \operatorname{codcch}=4005$

16. Stragliotto CL, Karlsson K, Lax I, et al. A retrospective study of SBRT of metastases in patients with primary sarcoma. Med Oncol. 2012;29(5):3431-3439. doi:10.1007/s12032-012-0256-2

17. Gunderson LL, Tepper JE. Clinical Radiation Oncology E-Book. Elsevier Health Sciences; 2011.

18. Spriestersbach A, Röhrig B, du Prel J-B, Gerhold-Ay A, Blettner M. Descriptive statistics. Dtsch Arztebl Int. 2009;106(36): 578-583. doi:10.3238/arztebl.2009.0578

19. Moher D, Liberati A, Tetzlaff J, Altman DG; PRISMA Group. Preferred reporting items for systematic reviews and metaanalyses: the PRISMA statement. PLoS Med. 2009;6(7): e1000097. doi:10.1371/journal.pmed.1000097

20. Kepka L, DeLaney TF, Suit HD, Goldberg SI. Results of radiation therapy for unresected soft-tissue sarcomas. Int J Radiat Oncol Biol Phys. 2005;63(3):852-859. doi:10.1016/j.ijrobp.2005.03.004

21. Zagars GK, Ballo MT, Pisters PWT, Pollock RE, Patel SR, Benjamin RS. Preoperative vs. postoperative radiation therapy for soft tissue sarcoma: a retrospective comparative evaluation of disease outcome. Int J Radiat Oncol Biol Phys. 2003;56(2): 482-488. doi:10.1016/s0360-3016(02)04510-8

22. Palm RF, Oliver DE, Yang GQ, Abuodeh Y, Naghavi AO, Johnstone PAS. The role of dose escalation and proton therapy in perioperative or definitive treatment of chondrosarcoma and chordoma: an analysis of the National Cancer Data Base. Cancer. 2019;125(4):642-651. doi:10.1002/cncr.31958

23. Rosenberg AE, Nielsen GP, Keel SB, et al. Chondrosarcoma of the base of the skull: a clinicopathologic study of 200 cases with emphasis on its distinction from chordoma. Am J Surg Pathol. 1999;23(11):1370-1378. doi:10.1097/00000478-19991100000007

24. Citrin DE. Recent developments in radiotherapy. $N$ Engl J Med. 2017;377(11):1065-1075. doi:10.1056/NEJMra1608986

25. Serizawa I, Kagei K, Kamada T, et al. Carbon ion radiotherapy for unresectable retroperitoneal sarcomas. Int J Radiat Oncol Biol Phys. 2009;75(4):1105-1110. doi:10.1016/j.ijrobp.2008.12.019

26. Greiner RH, Munkel G, Blattmann H, et al. Conformal radiotherapy for unresectable retroperitoneal soft tissue sarcoma. Int $J$ Radiat Oncol Biol Phys. 1992;22(2):333-341. doi:10.1016/03603016(92)90051-i

27. Kumar PP, Good RR. Interstitial 125 I implantation in the retreatment of retroperitoneal soft tissue sarcoma. Report of a case. Acta
Radiol Oncol. 1986;25(1):37-39. doi:10.3109/02841868609 136375

28. Yang B, Guo W-H, Lan T, et al. CT-guided 125I seed implantation for inoperable retroperitoneal sarcoma: a technique for delivery of local tumor brachytherapy. Exp Ther Med. 2016;12(6): 3843-3850. doi:10.3892/etm.2016.3897

29. Feng M, Murphy J, Griffith KA, et al. Long-term outcomes after radiotherapy for retroperitoneal and deep truncal sarcoma. Int $J$ Radiat Oncol Biol Phys. 2007;69(1):103-110. doi:10.1016/j. ijrobp.2007.02.041

30. Shelat VG, Pandya GJ, Shabbir A, Diddapur RK. Post radiation chylous ascites: a case report. Cases J. 2009;2:9393. doi:10.1186/ 1757-1626-2-9393

31. Akhavan A, Binesh F, Ghannadi F, Navabii H. Excellent response of malignant peripheral nerve sheath tumour of retroperitoneum to radiation therapy. BMJ Case Rep. 2012;2012. doi:10.1136/bcr2012-007266

32. Li Y, Wang Y, Liu B, Li Z, Wang W. 125I brachytherapy seeds implantation for inoperable low-grade leiomyosarcoma of inferior vena cava. Korean J Radiol. 2013;14(2):278-282. doi:10.3348/ kjr.2013.14.2.278

33. Sagara K, Takayoshi K, Kusumoto E, et al. Favorable control of rapidly progressive retroperitoneal pleomorphic leiomyosarcoma with multimodality therapy: a case report. BMC Res Notes. 2014; 7:377. doi:10.1186/1756-0500-7-377

34. Brenneman RJ, Sharifai N, Fischer-Valuck B, et al. Abscopal effect following proton beam radiotherapy in a patient with inoperable metastatic retroperitoneal sarcoma. Front Oncol. 2019;9. doi:10.3389/fonc.2019.00922

35. Xu-Holland A, Myburgh E, Donaldson W, Cranshaw I, Saran F. Feasibility of delivering high dose radical radiotherapy to retroperitoneal sarcomas. 2019 ASM PosterNG. Published 2019. Accessed May 2, 2020. https://posterng.netkey.at/ranzcr/view ing/index.php? module $=$ viewing_poster\&task $=\& p i=151886$

36. Ghanem AI, Glide-Hurst C, Siddiqui MS, Chetty IJ, Movsas B. Retroperitoneal metastasis abutting small bowel: a novel magnetic resonance-guided radiation approach. Cureus. 2018;10(4): e2412. doi:10.7759/cureus. 2412

37. Naghavi AO, Fernandez DC, Mesko N, et al. American Brachytherapy Society consensus statement for soft tissue sarcoma brachytherapy. Brachytherapy. 2017;16(3):466-489. doi:10.1016/ j.brachy.2017.02.004

38. Yoon SS, Chen Y-L, Kirsch DG, et al. Proton-beam, intensitymodulated, and/or intraoperative electron radiation therapy combined with aggressive anterior surgical resection for retroperitoneal sarcomas. Ann Surg Oncol. 2010;17(6):1515-1529. doi:10. 1245/s10434-010-0935-1

39. DeLaney TF, Chen Y-L, Baldini EH, et al. Phase 1 trial of preoperative image guided intensity modulated proton radiation therapy with simultaneously integrated boost to the high risk margin for retroperitoneal sarcomas. Adv Radiat Oncol. 2017;2(1):85-93. doi:10.1016/j.adro.2016.12.003

40. Imai R, Kamada T, Araki N; Working Group for Carbon Ion Radiotherapy for Bone and Soft Tissue Sarcomas. Carbon ion radiotherapy for unresectable localized axial soft tissue sarcoma. Cancer Med. 2018;7(9):4308-4314. doi:10.1002/cam4.1679 
41. Vitolo V, Barcellini A, Fossati P, et al. Carbon ion radiotherapy in the management of unusual liposarcomas: a case report. In Vivo. 2019;33(2):529-533. doi:10.21873/invivo.11506

42. Hayashi K, Yamamoto N, Shirai T, et al. Sequential histological findings and clinical response after carbon ion radiotherapy for unresectable sarcoma. Clin Transl Radiat Oncol. 2017;2:41-45. doi:10.1016/j.ctro.2017.01.002

43. Eckert F, Matuschek C, Mueller A-C, et al. Definitive radiotherapy and single-agent radiosensitizing ifosfamide in patients with localized, irresectable soft tissue sarcoma: a retrospective analysis. Radiat Oncol. 2010;5(1):55. doi:10.1186/1748-71 7X-5-55

44. Spalek M, Koseła-Paterczyk H, Borkowska A, et al. OC-0069 5x5 Gy with chemotherapy in borderline resectable soft tissue sarcomas: early results of a trial. Radiother Oncol. 2019;133:S31-S32. doi:10.1016/S0167-8140(19)30489-X

45. Nolan MW, Gieger TL, Karakashian AA, et al. Outcomes of spatially fractionated radiotherapy (GRID) for bulky soft tissue sarcomas in a large animal model. Technol Cancer Res Treat. 2017;16(3):357-365. doi:10.1177/1533034617690980

46. Wu X, Ahmed MM, Wright J, et al. On modern technical approaches of three-dimensional high-dose lattice radiotherapy (LRT). Cureus J Med Sci. 2010;2(3). doi:10.7759/cureus.9

47. Blanco Suarez JM, Amendola BE, Perez N, Amendola M, Wu X. The use of Lattice Radiation Therapy (LRT) in the treatment of bulky tumors: a case report of a large metastatic mixed Mullerian ovarian tumor. Cureus. 2015;7(11). doi:10.7759/cureus.389

48. Amendola B, Perez N, Amendola MA, et al. Lattice radiotherapy with RapidArc for treatment of gynecological tumors: dosimetric and early clinical evaluations. Cureus J Med Sci. 2010;2(9). doi: 10.7759/cureus. 15

49. Mohiuddin M, Memon M, Nobah A, et al. Locally advanced highgrade extremity soft tissue sarcoma: response with novel approach to neoadjuvant chemoradiation using induction spatially fractionated GRID radiotherapy (SFGRT). JCO. 2014;32(15_ suppl):10575-10575. doi:10.1200/jco.2014.32.15_suppl.10575 